Article

\title{
A Telemedicine Service System Exploiting BT/BLE Wireless Sensors for Remote Management of Chronic Patients
}

\author{
Massimiliano Donati ${ }^{1} * * \mathbb{C}$, Alessio Celli ${ }^{2}$, Alessio Ruiu ${ }^{2}$, Sergio Saponara ${ }^{1}$ and Luca Fanucci ${ }^{1}$ \\ 1 Department of Information Engineering, University of Pisa, via G. Caruso 16, 56122 Pisa, Italy; \\ sergio.saponara@unipi.it (S.S.); luca.fanucci@unipi.it (L.F.) \\ 2 IngeniArs srl, via Ponte a Piglieri 8, 56121 Pisa, Italy; alessio.celli@ingeniars.com (A.C.); \\ alessio.ruiu@ingeniars.com (A.R.) \\ * Correspondence: massimiliano.donati@unipi.it; Tel.: +39-050-2217-625
}

Received: 14 November 2018; Accepted: 15 January 2019; Published: 18 January 2019

\begin{abstract}
The management of the increasing number of patients affected by cardiovascular, pulmonary, and metabolic chronic diseases represents a major challenge for the National Health System (NHS) in any developed country. Chronic diseases are indeed the main cause of hospitalization, especially for elderly people, leading to sustainability problems due to the huge amount of resources required. In the last years, the adoption of the chronic care model (CCM) as assistive model improved the management of these patients and reduced the related healthcare costs. The diffusion of wireless sensors, portable devices and connectivity enables to implement new information and communication technology (ICT)-based innovative applications to further improve the outcomes of the CCM. This paper presents a telemedicine platform for data acquisition, distribution, processing, presentation, and storage, aimed to remotely monitor the clinical status of chronic patients. The proposed solution is based on monitoring kits, with wireless Bluetooth (BT)/ Bluetooth low energy (BLE) sensors and a gateway (i.e., smartphone or tablet) connected to a web-based cloud application that collects and makes available the clinical information to the medical staff. The platform allows clinicians and practitioners to monitor at distance their patients, according to personalized treatment plans, and to act promptly in case of aggravations, reducing hospitalizations and improving patients' quality of life.
\end{abstract}

Keywords: telemonitoring; telemedicine; wireless sensors; healthcare 4.0; cloud platform; Bluetooth (BT); Bluetooth low energy (BLE); Internet of Things (IoT); information and communication technology (ICT)

\section{Introduction}

Populations in industrialized countries are growing older and between one-third and one-half of total healthcare spending goes for the elderly in each country [1]. A significant part of such expenditure is related to the management of patients affected by the main chronic diseases: cardiovascular diseases, respiratory diseases and diabetes [2].

The prevalence of these chronic conditions is increasing among people of all ages, in particular in the older adults segment of population. Chronic diseases develop slowly, are long-lasting and often incurable. They are the main reason of death, cause great human suffering and place an enormous burden on National Health Systems (NHS) in terms of healthcare utilization and cost implications [3].

The World Health Organization (WHO) reports a prevalence of 251 million cases of chronic obstructive pulmonary disease (COPD) globally in 2016. COPD was the sixth leading cause of death worldwide in 1990 and recent studies assess that it will become the third in the next years, because of 
population aging and even because people is living longer and, therefore, being at risk of developing COPD for longer [4].

In 2015, more than 85 million people in Europe were living with cardiovascular diseases [5]. Among that, chronic heart failure (CHF) affects about $2 \%$ of the entire population in all industrialized countries. The fatality rate within the first year of disease is about $20 \%$ and becomes $50 \%$ within the first four years [6]. Statistics show that near $30 \%$ of patients are readmitted to hospital within 90 days from the first diagnosis. The readmission rate reaches $54 \%$ in the next six months.

According to $\mathrm{WHO}$, the number of people with diabetes has risen from 108 million in 1980 to 422 million in 2014 worldwide: the prevalence has risen from $4.7 \%$ in 1980 to $8.5 \%$ in 2014. Diabetes prevalence and incidence are foreseen to rise more rapidly than other chronic conditions.

In order to deal with this number of chronic patients, which is projected to increase rapidly in the following years, new and more effective healthcare models are required [7] to reduce the management costs charged to the NHS, to improve the patients' outcomes and to allow a better organization of the available resources.

A widely adopted approach to deliver healthcare services to chronic patients is the chronic care model (CCM) [8]. This model is based on personalized care and monitoring planning, on the presence of a multi-disciplinary care team (general practitioner, medical specialist, nurses, etc.), on the patient self-management of care, on the exploitation of domiciliary assistance and periodic visits. The latter are usually performed by professional caregivers. The CCM approach is based on the sharing of clinical information to support the decision making [9]. It is acknowledged in literature that the $\mathrm{CCM}$ improves the management of chronic conditions and reduces the costs incurred by healthcare systems [10]. Nevertheless, considering the increasing demand of care due to chronic conditions, new and scalable applications of the CCM are becoming necessary for clinicians and administrators.

In the age of Internet of Things (IoT), the advances of wireless sensors and mobile devices, along with the diffusion of short-range wireless technologies—such as Bluetooth (BT) and Bluetooth low energy (BLE), and Internet connectivity-allow to implement new ICT-based models for the management of chronic patients, enhancing the application of the traditional CCM [11]. The enabling technology for an effective implementation of such enhanced CCM is a telemedicine system that allows to collect all patients' vital parameters and signals through wireless sensors, and to share all relevant data in secure way among medical personnel in order to support the decision-making process with clinical evidence. Besides monitoring the health status, the same system can be used also to monitor the mood, the lifestyle and the psychological status of the patient.

This paper presents a flexible and highly configurable hardware/software telemedicine platform for data acquisition, communication, processing, presentation, storage and sharing; it aims to monitor at distance mood, lifestyle, vital signs and in general the clinical status of patients affected by chronic diseases. The proposed platform meets all the requirements of the traditional CCM and allows a scalable provisioning of healthcare monitoring services, according to personalized care planning.

The present work represents an extension of the paper presented at the conference IEEE MOCAST 2018 by the same authors [12]. In particular, this article includes a detailed explanation of the motivation of the work and an extended the state-of-the-art analysis, provides additional details regarding the telemedicine gateway and the centralized server application, and finally discusses the impact of the system in the chronic patient's management.

Hereafter, Section 2 reports the comparison of the proposed system vs the state-of-the-art. Section 3 presents the architecture of the whole hardware/software system. Section 4 deals with wireless sensors and monitoring kit. Section 5 presents the development of the server application and repository. The impact of the adoption of the proposed solution on the health care systems and on the medical staff every day work is discussed in Section 6. Conclusions are drawn in Section 7. 


\section{Comparison with the State-of-the-Art}

In the state-of-the-art, a lot of research works present innovative, wearable or non-invasive, medical sensors [13-20] with wireless capability, while a very large set of wireless devices (e.g., blood pressure monitor, thermometers, etc.) are already available on the market as COTS components. These sensors, and the promising research prototypes, can be integrated in the proposed platform, with a minimal development effort, to extend its features.

Considering telemedicine systems for chronic patients in the research literature [21-25] or present on the market [26-28], it is possible to note that they often provide only a partial support of scenarios suitable for the out-of-hospital monitoring of chronic patients. Instead, the proposed system includes on the same service platform all actors (i.e., care team), all relevant data in integrated way and all the possible scenarios involved in personalized healthcare service provisioning. In particular, the proposed solution meets three main telemedicine scenarios: (i) the patient self-acquisition scenario (i.e., one specific monitoring kit for each patient); (ii) the doctor's visit scenario (i.e., a dedicated monitoring kit operated by professional caregivers to manage a set of patients); and (iii) the point of care scenario (i.e., specific acquisition equipment hosted in specific building like a pharmacy or a residence for elderly people). A patient can be treated with a combination of different scenarios depending on his/her conditions. Within each scenario, kind and frequency of data acquisition can be configured according to the patient's needs; and the acquired data are immediately made available to the patient's care team members.

The flexibility and configurability of the presented platform make the solution suitable to monitor patients affected by one or more generic chronic conditions, which outcomes benefit from a frequent assessment of the clinical status based on vital signs and lifestyle parameters. Conversely, most of the systems in the state-of-the-art are specific for the monitoring of a specific disease (e.g., chronic heart failure, etc.).

Finally, the proposed system exploits dedicated levels of user interface for each actor: from the easy to use interfaces dedicated to elderly people involved in the self-monitoring scenario to the advanced and complete interfaces for professional operators. In all cases, the rationale is to provide in effective way the user-specific features of the system, minimizing the effort required to understand and use them. This user-centric customization of the system interfaces is not always taken into account in other works published in literature, especially for that concerning the patient-oriented interfaces.

\section{Hardware/Software System}

The proposed system is composed by several monitoring kits distributed on the territory, precisely at patients' home, and a centralized server software application with storage capabilities, usually installed in the service provider facility. The bi-directional communication among these subsystems goes through the Internet network, exploiting broadband communication technologies (i.e., 3G, 4G, $5 \mathrm{G}$, or WiFi connection to existing access-points). The overall client-server architecture of the system is shown in Figure 1.

The monitoring kit is organized as an IoT sensors network, which uses BT/BLE as short-range communication technology [29]. It includes a set of non-invasive wireless sensors [30] and an Android device (i.e., tablet or smartphone), called gateway [31], equipped with a dedicated software application in charge of managing acquisition, local storage and transmission of physiological data and lifestyle parameters gathered automatically from the sensors or provided by the user. 


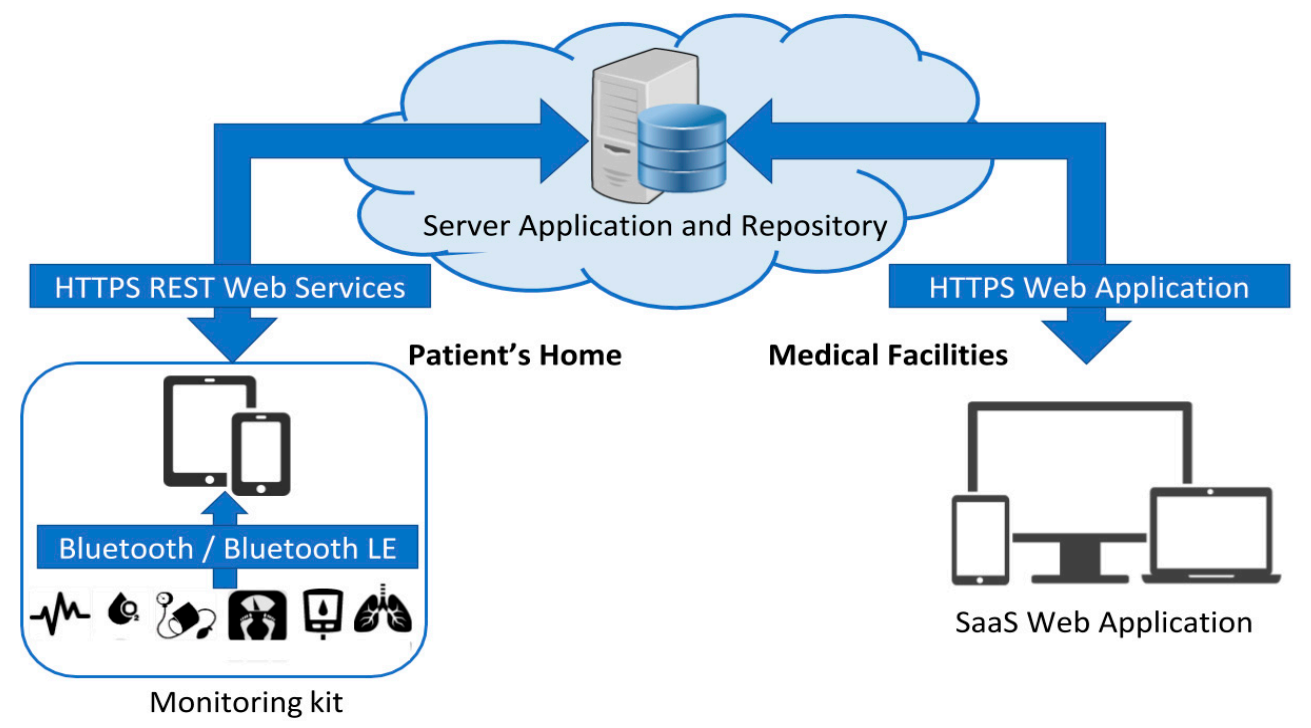

Figure 1. Architecture of the hardware/software system.

The server application represents the centralized node of the system. This cloud-oriented software provides the resources for the communication, elaboration, graphical presentation, and management of all the data related to the patients enrolled in the telemonitoring service. The server application exposes some Representational State Transfer (REST) web services that allow the gateways to upload acquired data and to receive configuration updates remotely (e.g., personalized care plan of the specific patient, etc.). Additionally, it runs algorithms for data analysis and alarm situations detection. The packets exchanged among the server and the gateways use a proprietary set of XML tags, while standard HL7 Clinical Document Architecture (CDA) [32] is used for the integration with external third-party software and information systems (e.g., other clinical records, hospital information systems, etc.). For the user, the server offers a multi-access multi-profile web application according to the Software as a Service (SaaS) paradigm. Such a web-based application enables medical staff to visualize and manage all data concerning the patients' care (e.g., vital signs, parameters, personalized care plans, generated alarms, etc.) exploiting the browser of personal computers or mobile devices.

The repository, implemented via a relational database, handles the persistence of the patients' electronic health records (EHR). It provides long-term storage of data acquired through the monitoring kits, data generated by the platform algorithms and data manually inserted by the medical staff during the monitoring period.

Both the web services and the web application use HTTPS protocol and basic authentication of the user to enforce confidentiality, authenticity, and integrity of the communications flows over the public network.

\section{Monitoring Kit and Wireless Sensors}

The monitoring kit is the part of the proposed telemedicine system that allows the acquisition and transmission of vital signs, lifestyle parameters and other information about the patient status, according to a care plan tailored on him/her specific needs. This sensor network includes a gateway device and a set of BT/BLE biomedical sensors.

The gateway is the central node of the sensor network. It coordinates the bi-directional communications with the BT/BLE sensors to receive measurement data, either if they are master or slave in connection establishment. Moreover, the gateway handles the request-response communications with the server application exploiting WiFi or mobile broadband connectivity (e.g., $3 \mathrm{G}, 4 \mathrm{G}$, etc.). It connects to the server application both to download the specific care plan established by clinicians and to deliver acquired data in secure way. In particular, the care plan is a list of actions/activities (e.g., measurements, questionnaires, drugs assumptions, etc.) characterized by 
request time and deadline, that clinicians define and update in accordance to the specific patient needs. Basing on this care plan, the gateway requests to perform the activities and elaborate the resulting data. All these results are finally sent to the server application, making them available to the medical staff that monitor at distance the patients' status.

In order to guide the user to follow the activities defined in the personalized care plan, the gateway also features an intuitive and smart graphical user interface focused in minimizing and simplifying the interactions with the system.

The set of supported sensors comprises several non-invasive, BT/BLE wireless, medical-qualified devices for the measurement of the main vital signs. The choice of BT/BLE sensors has been made because of the high diffusion on the market of a wide range of devices adopting this communication technologies. Currently, the gateway is able to communicate with the commercial medical devices listed in Table 1. The specific sensor group is selected for each patient according to the kind and severity of the disease and may change during the monitoring period.

Table 1. List of COTS devices integrated in the system.

\begin{tabular}{|c|c|}
\hline Measurement/Exam Device & COTS Sensor \\
\hline ECG (Electrocardiography) device & $\begin{array}{l}\text { CARDIOLINE Microtel Cardiette } \\
\text { CORSCIENCE COR12 } \\
\text { CONTEC ECG Palmtop PM-10 }\end{array}$ \\
\hline Blood pressure monitor & $\begin{array}{l}\text { AND UA-767pbt } \\
\text { AND UA-767pbt-Ci } \\
\text { AND UA 651BLE } \\
\text { TRANSTEK LS802-B } \\
\text { TRANSTEK LS805-B } \\
\text { TAIDOC 3128-B }\end{array}$ \\
\hline Oximeter & $\begin{array}{l}\text { NONIN ONYX II 9560BT } \\
\text { NONIN } 3240 \\
\text { GIMA OXY } 10 \\
\text { TAIDOC } 8255\end{array}$ \\
\hline Spirometer & $\begin{array}{l}\text { MIR Spirotel } \\
\text { MIR Spirodoc } \\
\text { CONTEC Spirometer SP-10W } \\
\text { VITALOGRAPH COPD-6 }\end{array}$ \\
\hline Digital scale & $\begin{array}{l}\text { AND UC 351pbt-Ci } \\
\text { AND UC 352BLE } \\
\text { TAIDOC 2555-B } \\
\text { TRANSTEK Body Fat LS-203 }\end{array}$ \\
\hline Thermometer & $\begin{array}{l}\text { FORACARE IR20b } \\
\text { TAIDOC 1261C } \\
\text { TAIDOC } 1241 \mathrm{~B}\end{array}$ \\
\hline Glucometer & $\begin{array}{l}\text { LIFESCAN OneTouch Ultraeasy } \\
\text { CONTEC Blood Glucose Meter } \\
\text { TAIDOC 4279-B } \\
\text { GIMA ON-CALL Plus }\end{array}$ \\
\hline
\end{tabular}

Besides the vital signs and signals measured by these medical-qualified devices, the gateway is also able to collect lifestyle parameters related to physical activity (i.e., steps count, physical activities tracking, calories intake and consumption) from different kinds of wireless BT/BLE devices, such as the bracelets usually employed in personal wellness applications.

Since BT/BLE technology operates in the same frequency range of the WiFi (i.e., around $2.4 \mathrm{GHz}$ ), possible interference problems can occur [33]. This problem is mitigated by the adaptive frequency hopping (AFH) mechanism [34] that is currently part of the BT/BLE stack. Other WiFi frequencies are 
around $5 \mathrm{GHz}$, and mobile broadband standards use a different frequency range. For these reasons, electromagnetic interference does not account in significant way the system functionality.

Another class of data collected by the gateway comes from custom or predefined questionnaires that the patient could be requested to answer during the monitoring period. Questions are proposed one by one to the patient, giving him/her to possibility to answer using the gateway touchscreen. The gateway supports both open answer and selectable answer questions. For example, this feature allows to get subjective evaluations of the level of stress or the health feeling perceived by the patient.

The general architecture of the gateway software application is shown in Figure 2. In particular:

- the acquisition module handles the I/O operations via sensor-specific drivers activated on-demand by a dedicated manager;

- the storage module manages the persistence of all collected data into a local database, implemented with the Android native database management system (DBMS) SQLite;

- the network module manages the bi-directional communication with the server application (i.e., upload of data acquired by the biomedical devices and download of configurations and patient care plan);

- the user interface module manages the application front-end (e.g., screens, audio-visual feedbacks, user interactions, etc.);

- the core module is responsible of the patient care plan activities scheduling, data processing, and coordination of all the application tasks.

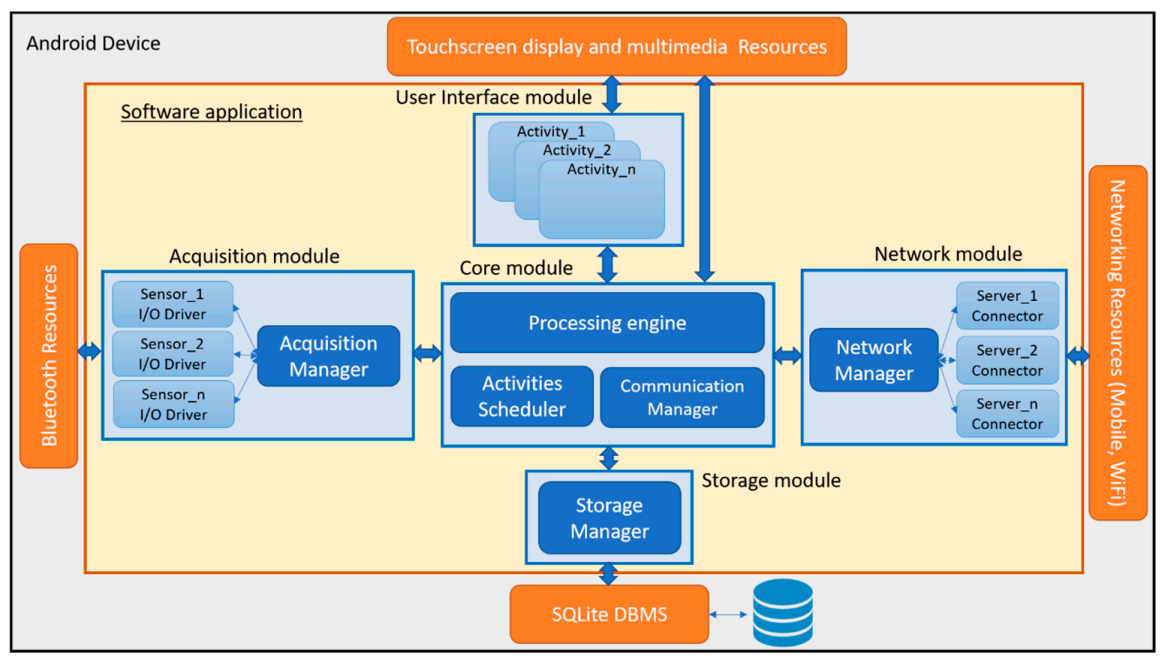

Figure 2. Architecture of the gateway software application.

Two kinds of monitoring kit have been developed. The first is dedicated to patient self-monitoring and is usually delivered directly to the patient when enrolled in the telemedicine service. The second is designed to support medical operators during the planned periodic home visits. They differ in the version of the gateway and usually in the set of sensors included in the kit, that depends on the target user and the specific disease to be monitored.

The two types of gateway rely on different software applications. They have the same software architecture and mainly differ for the implementation of the user interface module. Both versions have been implemented with the native Android SDK and are compatible with commercial smartphones and tablets with operating system (OS) version $\geq 4.3$. The applications require devices equipped with dual-mode Bluetooth chipset, network connectivity (i.e., Wi-Fi and mobile broadband) and at least 4 GB of storage memory for local storage of data. In terms of processing power and RAM memory, they do not require any resource in addition to those required by the Android OS version running on the chosen device. 
The patient-oriented gateway (see Figure 3) is conceived to maximize the usability and to minimize the impact on the patient. It enables the patient to follow autonomously the personalized treatment plan, defined and updatable by the care team members via the server application, thanks to audio-visual reminder messages and helpers. The data acquisition, elaboration, and transmission are completely automatic and transparent. Moreover, the gateway is able to collect out-of-plan activities performed on a personal basis.

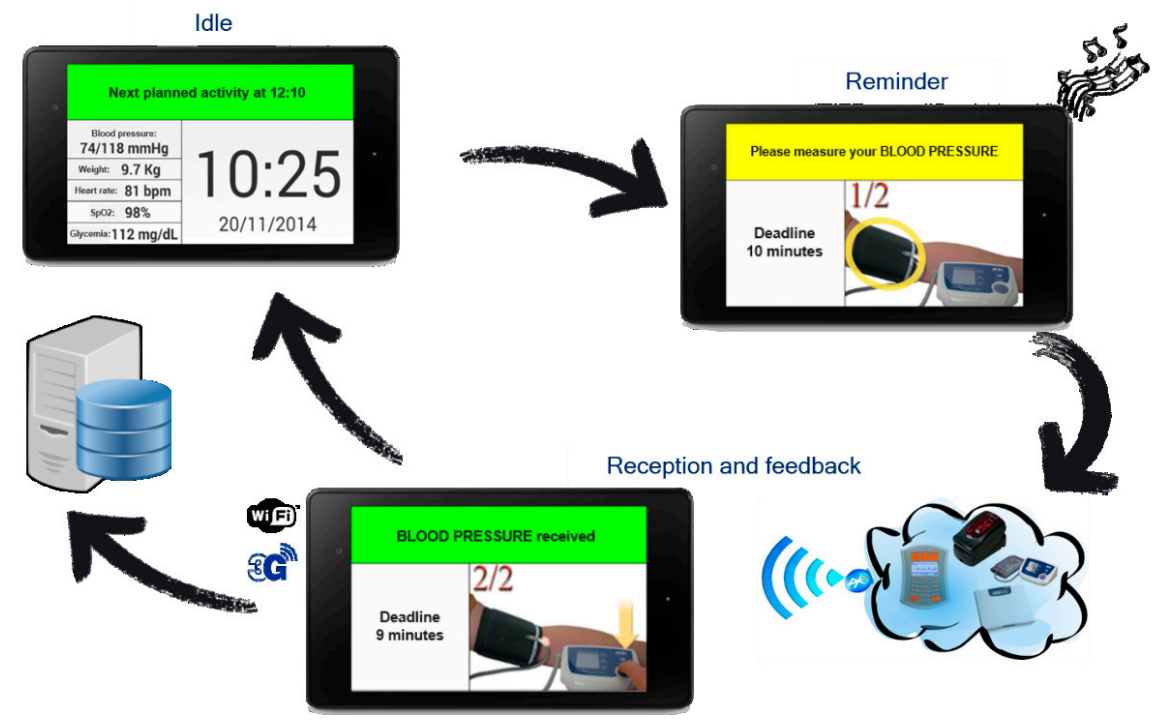

Figure 3. Example of blood pressure acquisition and transmission with the patient-oriented gateway.

The professional-oriented gateway (see Figure 4) supports assistance operators and simplifies their work during routinely domiciliary visits of chronic patients. It allows the management of the large list of patients (e.g., 50 or more), specifying for each of them the personalized plan of activities to be performed. This gateway extends the functionality of the previous version including more sophisticated data viewers and interaction modes suitable for the professional user (e.g., signals and trend lines viewers, manual recording of measures, etc.).

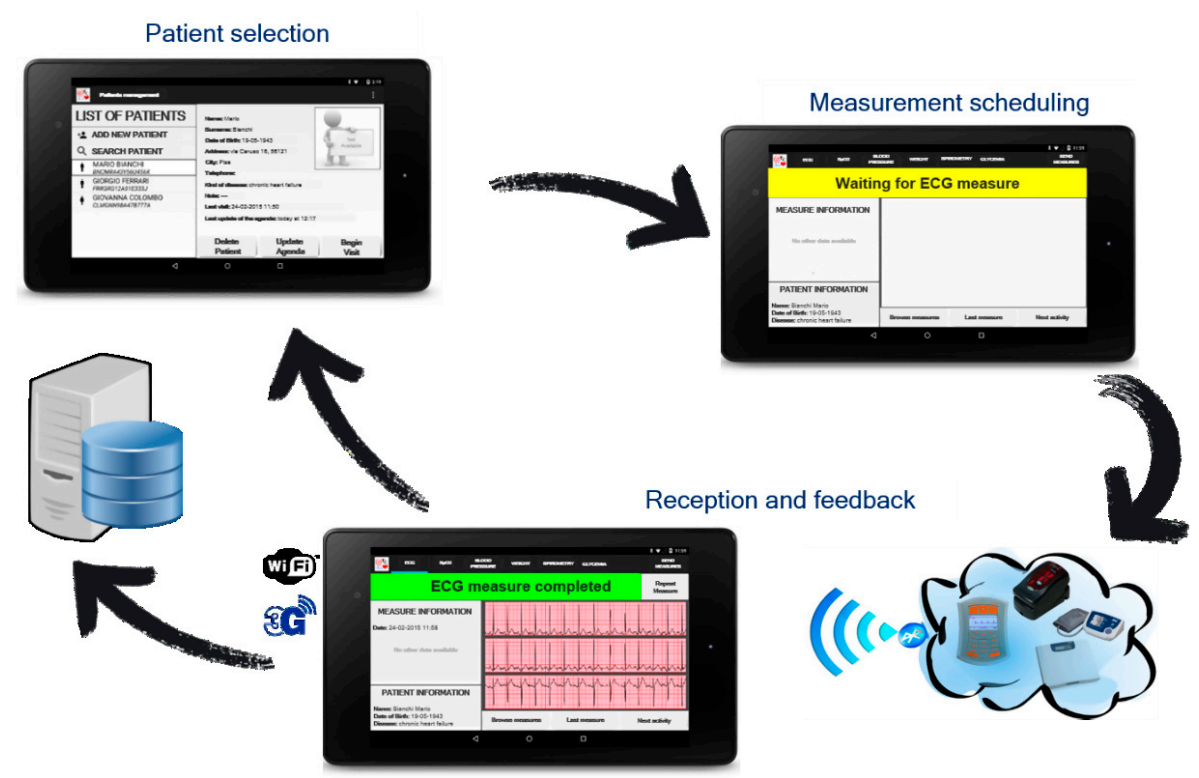

Figure 4. Example of Electrocardiography (ECG) acquisition and transmission with the professionaloriented gateway. 


\section{Server Application and Repository}

The central element of the proposed telemedicine system is a cloud-oriented application with web-based user interface, web services and repository capabilities, that allows the complete management of the telemonitoring service operated by medical personnel.

In particular, this server software application is in charge of:

- managing the bi-directional communication with the gateways to receive collected data and send updated care plans;

- providing web-based graphical user interface to present the monitoring data of the patients, to update the care plans, to define alarm detection rules, to manage raised alarms, to enroll new patients, etc.;

- highlighting missing data and analyzing incoming data to detect potentially dangerous situations for the patients in case of specific detection rules have been defined;

- synchronizing data with the external repositories and other third-party information systems;

- interfacing with the local repository to store and retrieve data.

The repository provides long-term storage of the EHR of the monitored patients. For each patient, the EHR contains at least the registry information, the data received from the gateway, the alarms generated by the analysis algorithms, the care plan and the alarm detection rules. Optional recordable data are about the characterization of the class and severity of the main affecting disease, eventual presence of co-morbidities, medical reports, annotations, etc. Additionally, it tracks all the changes performed by medical staff over the time.

In general, the server application offers its functionalities exposing web-based endpoints (i.e., URLs). All the incoming requests are firstly authenticated, then dispatched to the interested domain logic modules in order to process the contained data and to produce the response for the client.

The modular architecture of the server software application and its relation with the local repository is shown in Figure 5. The functionalities of the main blocks are described hereafter.

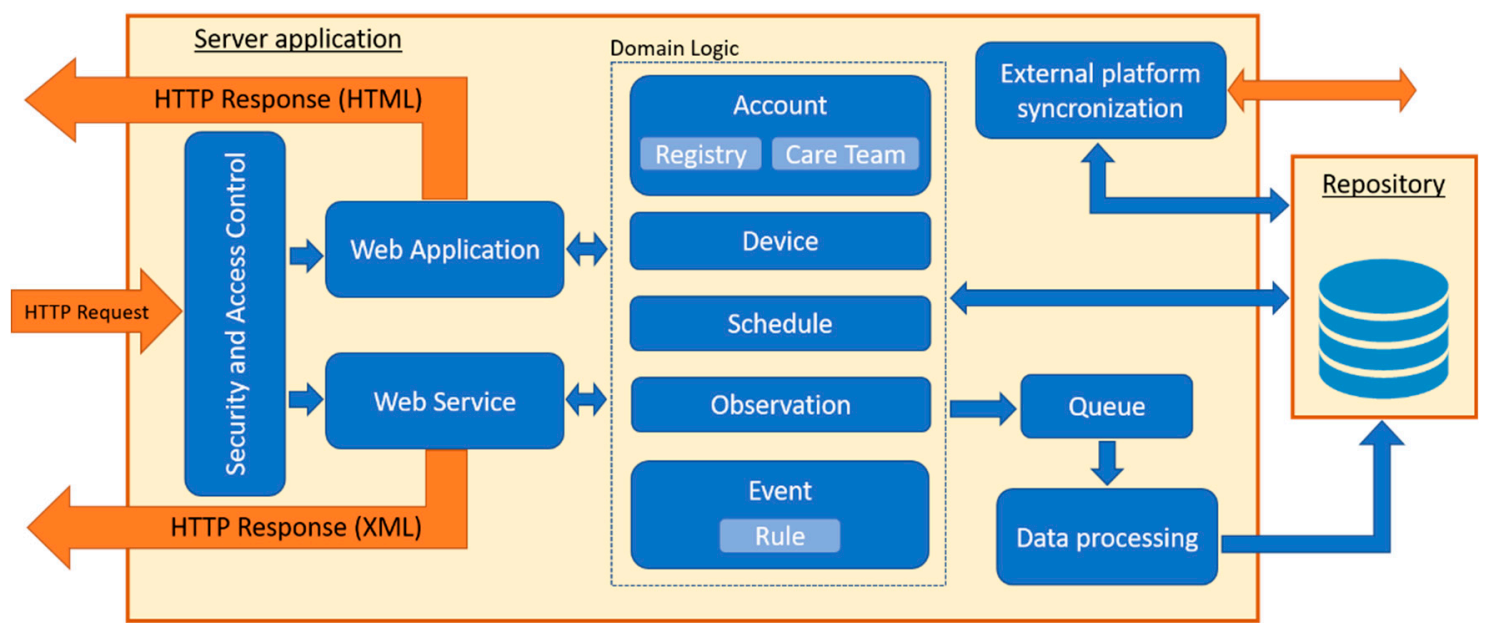

Figure 5. Architecture of the server software application and repository.

The security and access control module receives the HTTP requests over an SSL connection (i.e., HTTPS) from the clients and manages the authentication according to the basic strategy (i.e., username and password). Moreover, it restricts the access to the resources basing on the role of the logged user (i.e., general practitioner, medical specialist, nursing operator, and patient).

The web application module handles the graphical user interface (GUI) endpoints. The GUI is available via a common web browser, according to the SaaS cloud paradigm. It consists of several web pages based on HTML5, CSS, and JavaScript that the user can request to insert new data into the 
repository or consulting the stored data. Web pages are designed to adapt on personal computer and mobile devices screen form factors.

The web services module manages the endpoints that allow the gateway to exchange contents with the server application according to the REST paradigm. This module uses a custom XML schema for the request-response messages validation. Operations that requires data writing into the repository (e.g., upload of data collected by the gateway, etc.) are buffered through a FIFO queue and served asynchronously by the data processing module. Instead, query requests for data are immediately served (e.g., download of patient care plan, daily activities, summary information, etc.).

The data processing module is in charge of elaborating the data extracted from the queue in order to store them permanently into the repository. These data are observations results (e.g., vital signs, lifestyle parameters, questionnaires, etc.) and alarms generated by the analysis algorithms.

The synchronization module allows the integration and the interoperation of the platform with external information systems and applications (e.g., hospital information systems, repositories, medical records, etc.), in order to import/export data. It uses HL7 CDA standard format [35].

The domain logic consists of several cooperating modules providing the application-specific features. In particular:

- The Account module handles the users accounts (e.g., usernames, passwords, user roles, account expirations, etc.) and ensures the compliance to the General Data Protection Regulation (GDPR). This module is strictly connected to:

the Registry sub-module, that manages all the users personal data (e.g., profiling data, users contacts and availability places);

- the Care Team sub-module, that keeps track of the relations among accounts of patients and medical personnel involved in their care, in order to deliver patient information only to enabled medical staff.

- The Device module manages the list of devices (i.e., gateways and sensors) enabled to communicate with the server application.

- The Schedule module provides the functionality for the care plan assignment, editing, and consultation.

- The Observation module is in charge of the management of vital signs, signals, lifestyle, and questionnaire data of the monitored patients.

- The Event module takes care of the events/alarms detection and management. It uses specific algorithms and patients' personalized thresholds to find critical situations or dangerous alterations of vital signs and signals. The specific rules, set up by medical staff, are managed by the Rule module.

The described architecture has been implemented with Java Enterprise Edition (JEE) using also Spring Web and Spring Security frameworks. The repository has been implemented MySQL DBMS. The software application is compatible with application servers such as Apache Tomcat.

Concerning the web application GUI, Figure 6 shows the clinicians-specific landing page, presented by the system immediately after a successful login procedure. This page highlights the patients that needs immediate attention, providing for each of them the highest-level pending alarm to be managed. Moreover, the page enables the user to easily access to the functionalities offered by the system. The main functionalities are further described in the following subsections. 


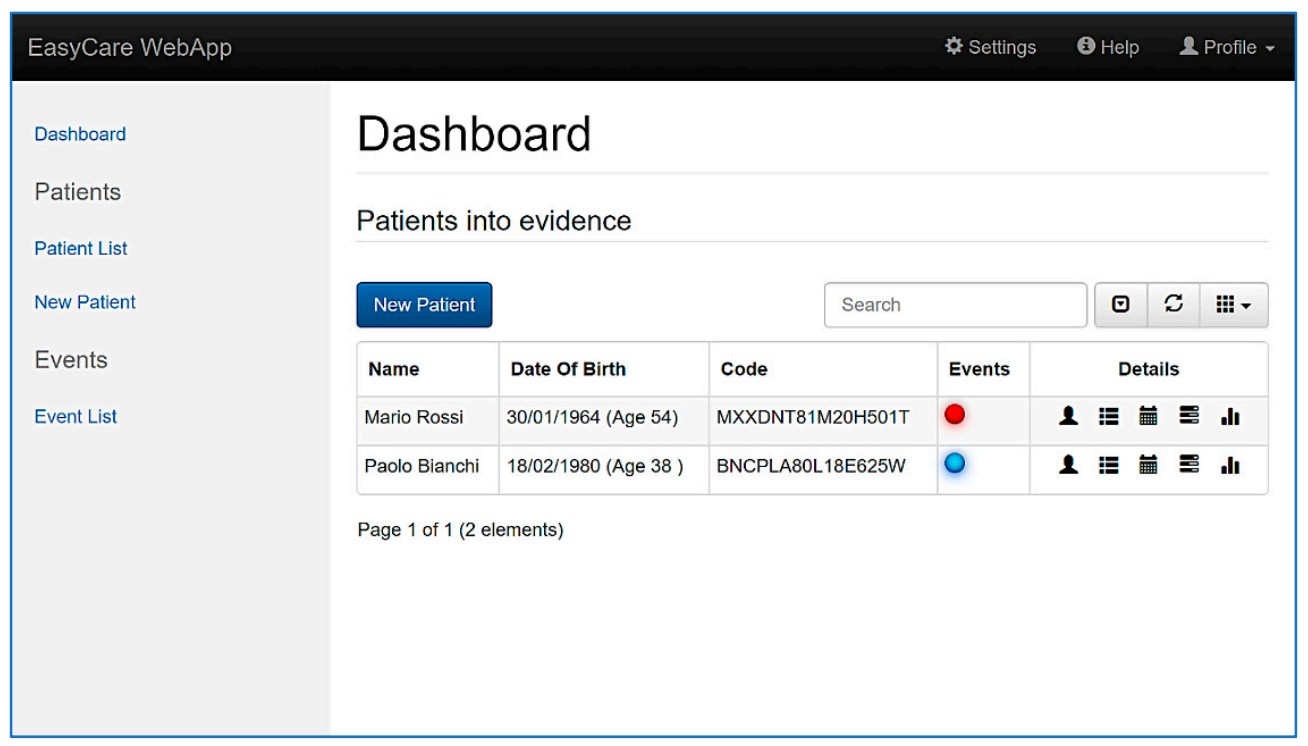

Figure 6. Dashboard page for clinicians.

\subsection{Patient Enrolment}

The server application offers to the medical staff the possibility to register new patients via an HTML form, adding a patient's registry information along with his/her contacts and availability places. The application automatically creates an account associated to the new patient, by means of which the patient is enabled to log in the application and consult his/her own data.

At the same time of the registration, a reference physician is associated to the patient, creating this way the initial care team of the patient. The possibility to add other care team members is given to the reference physician, allowing other doctors to access patient data and help to make diagnosis or modify the care plan.

During the enrolment phase, the medical staff informs the patient about the privacy terms of services regarding his/her data management processes.

\subsection{Care Plan Management}

The care plan management feature offers to the care team the possibility to schedule periodic monitoring activities of specific vital signs. Figure 7 shows the GUI page dedicated to the care plan management, were a complete perspective of the activities involving the patient is offered to the user.

The care plan is available for the patient via the server application or the gateway application, and it guides the patient in the care plan compliance by means of a reminder mechanism.

At the same time, the application offers to care team members the possibility to have some specific notifications in case of some event/alarms happens carrying out the activities. 


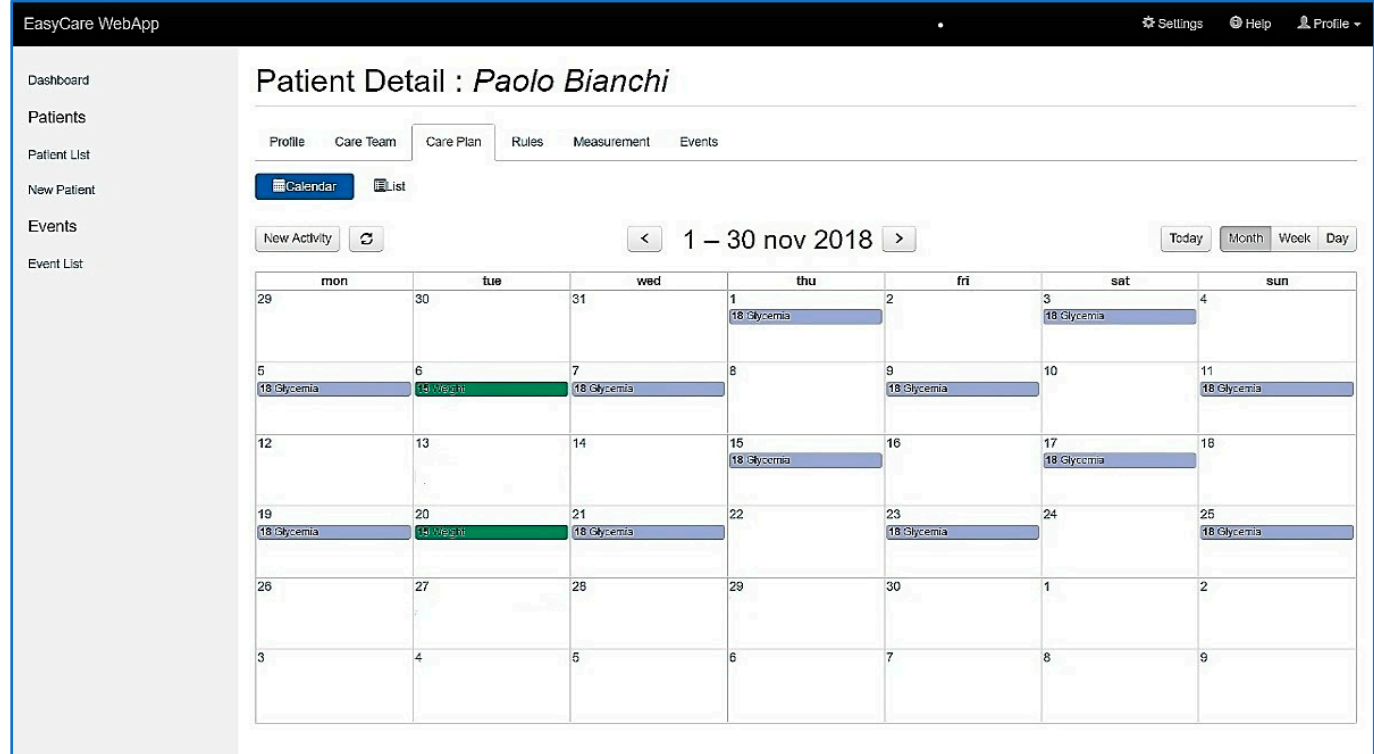

Figure 7. Patient-specific care plan management page.

\subsection{Measurements Consultation}

The application user interface enables care team members to monitor vital signs, signals, lifestyle parameters and questionnaire answers coming from patients' activities by means of specific reporting pages. As an example, Figure 8 shows a chart that reports the seven-day trend of the measurements of the sugar level in blood.

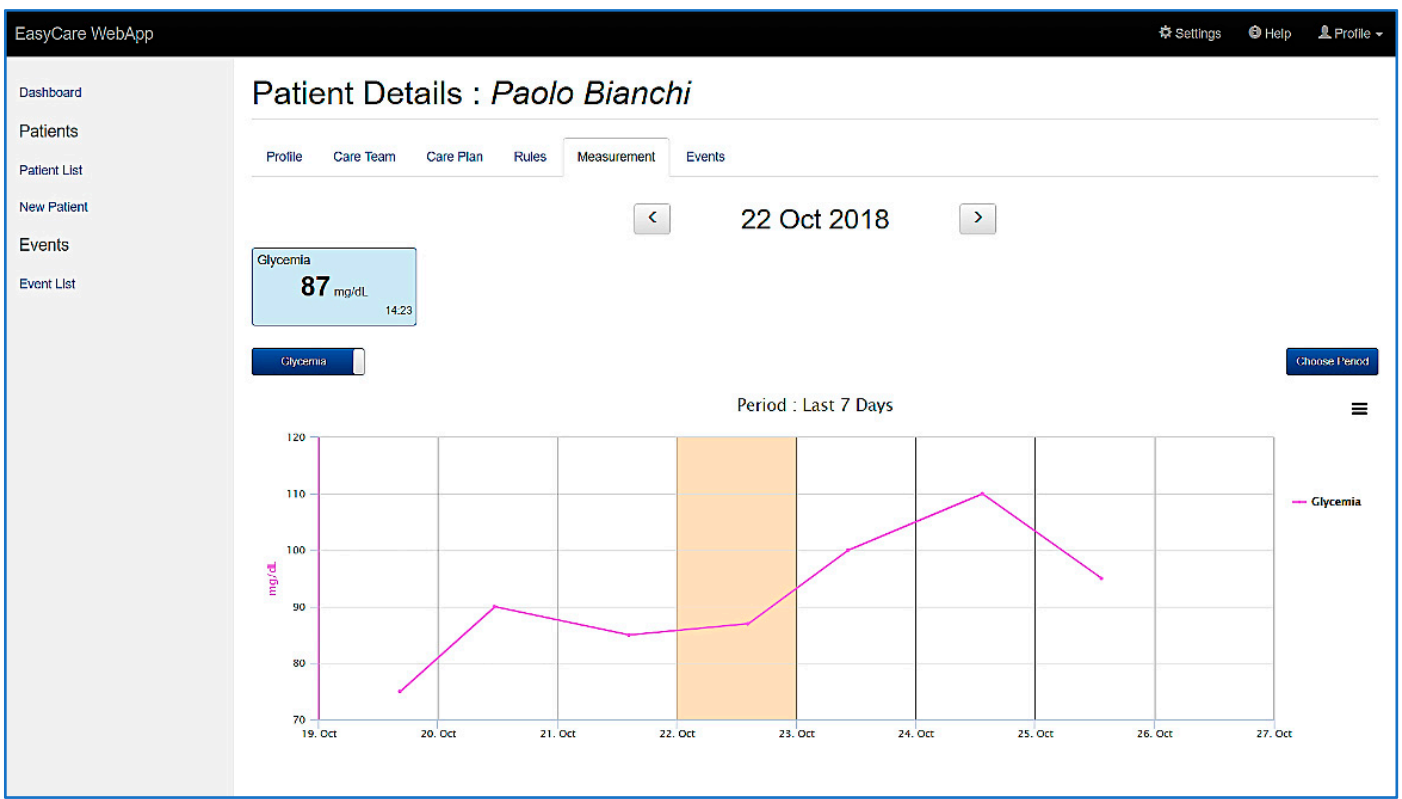

Figure 8. Patient-specific measurement results chart.

Via the chart, the care team member can analyze the temporal evolution of a specific parameter, thus inferring the status of a patient. The charts can display information for custom periods, depending on user needs, and permit them to select a specific day of the care plan to display measurements carried out by the patient on that day. For each point of the chart it is possible to obtain detailed information (i.e., acquisition date, values, and connected alarms if present). 
This feature implements a very flexible and complete dashboard available to professional users to monitor the trend and have a complete picture of the patient health status, helping them in making decisions based on clinical evidences.

\subsection{Events and Alarms Management}

Events and alarms are raised by the algorithms running on the server application when one of the following situations occur:

- a measurement violates a threshold set up by a care tam members;

- a scheduled measurement do not arrive to the server application within its deadline;

- a non-scheduled measurement arrives to the server application.

They are raised independently for each patient if and only if a physician set a dedicated rule (i.e., threshold violation, missing activity, extra protocol activity) associated to a specific activity, vital signs or parameter, thus enabling the care team to decide when an event has to be raised.

The application gives the possibility to a care team member to take in charge and resolve events and alarms by means of a specific page, shown in Figure 9. This page also presents information about the event/alarm context (e.g., the violated rule, the interested parameter history, etc.).

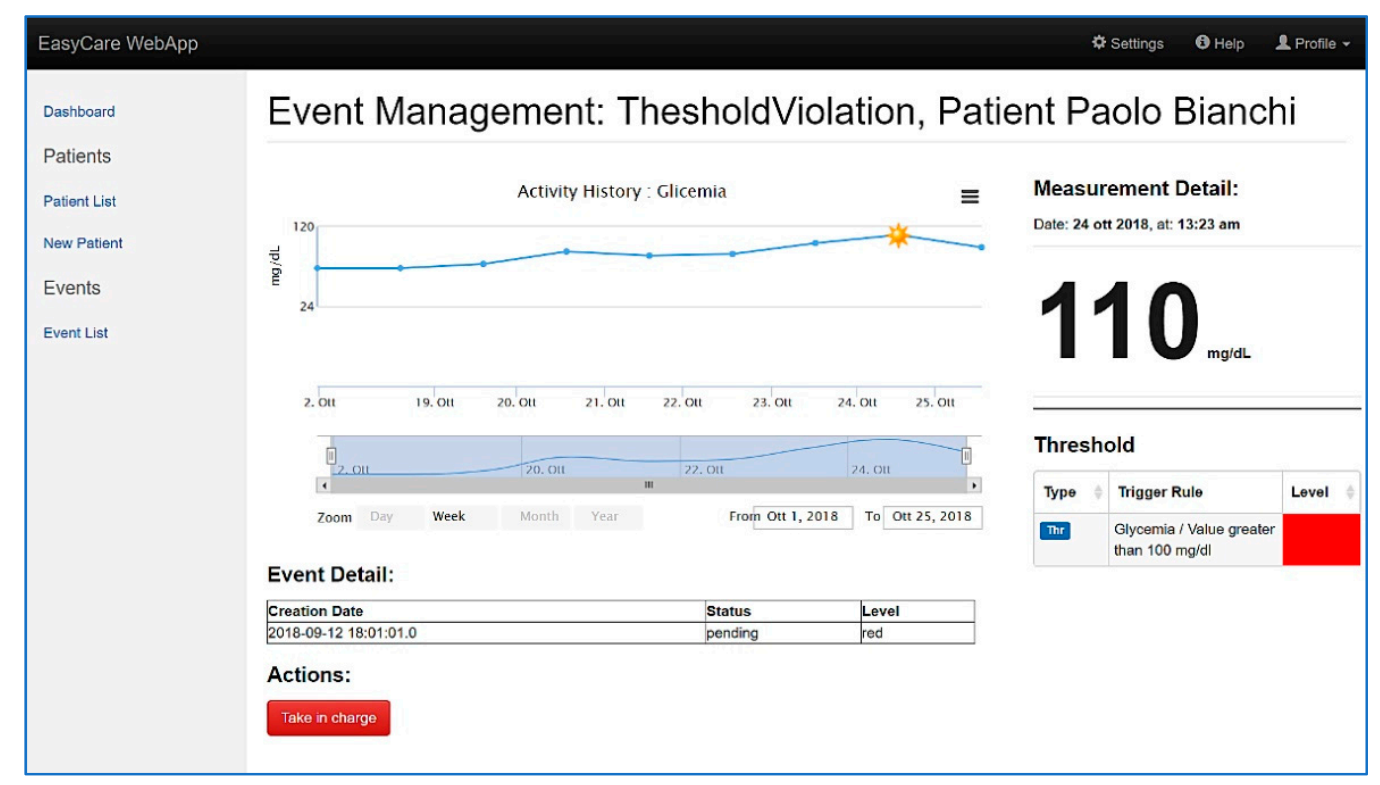

Figure 9. Event and alarm management page.

\section{Discussion}

In recent years, sectors such as industry, logistics, and administration have experienced a real revolution, thanks to the enhancements introduced by ICT solutions employment. This allowed to find smart and innovative solutions to well-known problems and to improve the effectiveness of the supplied services. More recently, the diffusion of mobile connectivity, the enhancement of the wireless sensor networks and the development of the IoT paradigm made possible to acquire and distribute an ever increasing amount of monitoring data.

The system described in this work represents an application of these technologies to face the sustainability problem and to improve the outcomes regarding the management of chronic diseases. While the traditional health care model (i.e., CCM) is getting inadequate for the increasing number of patients, the aim of the proposed system is to fulfil the needs of continuous monitoring of patients, improving the treatment methods according to a more efficient and proactive model of care vs the reactive model (i.e., management of emergency situations) and the traditional application of the CCM. 
This Healthcare 4.0 service platform, developed to support a new ICT-enhanced application of the CCM, can overcome the limits of the traditional model of care that affects both the patients' quality of life and the resource utilization of NHS. The employment of the proposed platform allows the complete digitalization of all the CCM processes and the sharing of clinical information among care team members, without time and distance barriers, to support diagnosis and decision making. More precisely, the platform introduces a very high optimization of the chronic patient management processes, enabling the care team members to remotely assign personalized care plans and monitor at distance patients' health status without the need of frequent domiciliary or in-hospital visits. It can obtain a shared and integrated data flow, with objective and subjective data about the patient's status, from the automated acquisition (i.e., sensor data or questionnaire) to the final user (i.e., care team member); and this data flow can be further integrated with existing information systems to provide a wider sharing of clinical information. Errors due to manual transcription of vital parameters and delay in information sharing are avoided, improving the effectiveness of the telemedicine service. Furthermore, the configurability of the system allows the care team to tailor the service on the specific patient's needs and to make adjustment easily during the monitoring period.

The availability of both objective data, coming from sensors, and subjective data, provided by the patient himself/herself (i.e., questionnaires), allows a complete and frequent assessment of the health status based on multi-dimensional evidences, otherwise impossible with the traditional CCM due to lack of resources and the increasing incidence of chronic conditions.

In order to deal with this large amount of monitoring data and to optimize the time that medical staff needs to take care of patients, the system provides a very efficient and flexible mechanism of data visualization, data analysis, configuration and alarm situations management. In particular, it provides a very intuitive and complete set of web pages to medical users to give aggregated and patient-specific pictures of the health status with charts, trend lines, and historical data. Moreover, the system allows to set and update the list of measurements and the rules triggering alarms of different priorities specifically for each patient. This flexibility in configuring the monitoring profile enables to meets the patient-specific needs and also to make changes required due to the evolution of the disease. The system is also able to automatically highlight the patients that need attention because they have pending alarms related to measured vital signs, so that the care team members can act promptly when an aggravation is detected. The platform ensures that all care team members view the same clinical information about the patient and be aware of the operations performed by the other members. In addition, every member can include other physicians or specialists in the care team, enabling them to consult patients' data and further simplifying the process of asking consultations and second-opinions.

In order to apply this ICT-enhanced CCM in the daily practice, the patient's existing care team members receive a specific credential to access the system and to manage the assisted patients, that in turn are provided with an appropriate monitoring kit when enrolled in the service. In particular, thanks to the web-based user-interface, all data are available in every place the care team member has an internet connection and a web browser at disposition (e.g., at medical practice, at home, in mobility, etc.). This enables each member to take advantage of the system features in every-day work, according to his/her role, not only to monitor remotely the evolution of the chronic disease but also to improve the management of other medical conditions.

\section{Conclusions}

The paper has presented a telemedicine service platform which allows an effective and integrated application of the chronic care model (CCM) for the out-of-hospital treatment of patients affected by chronic conditions. Indeed, at the state-of-the-art the CCM is not often implemented through a fully integrated ICT-based information system, with a seamless digital data flow from the sensors used by the patient to the desktop of the remote caregiver.

The platform exploits monitoring kits that includes wireless sensors with BT/BLE connectivity to the local gateway (i.e., tablet/smartphone with dedicated software) and a connection to a centralized 
cloud-oriented application for data processing and storage (i.e., repository). The monitoring kit is available both in patient-oriented and professional-oriented versions. The server SaaS application allows the care team members to manage the enrolled patients and to monitor at distance their vital, emotional, and psychological parameters according to personalized care plans. The aim is continuously supporting the medical staff with updated data, thus moving from a medicine of emergency to a scalable and proactive healthcare model. Furthermore, the system generates alarms on vital signs thresholds violations and performs multi-parameters analysis, enabling the medical staff to promptly realize aggravations before they become irreversible and lead to hospitalization. In this way it is possible to reduce the hospitalization rate, and hence the related cost for the NHS. Moreover, this model allows an optimization in the allocation of the medical resources (e.g., personnel time, facilities, visits, etc.). Outside the hospital, the quality of life of the patient will be improved as well as the mortality rate decrease.

Finally, in order to extend the platform capabilities, some additional features such as medical supersession and specific algorithms based on artificial intelligence (AI) for risky and dangerous situations prediction are under development and will be included in the next release of the system.

Author Contributions: The contribution of all authors was equal.

Funding: This research was partially funded by Regione Toscana AIRCARDIO project (POR CreO FESR 2014-2020 action).

Acknowledgments: We acknowledge discussion with the AIRCARDIO project team.

Conflicts of Interest: The authors declare no conflict of interest.

\section{References}

1. Anderson, G.F.; Hussey, P.S. Population Aging: A Comparison Among Industrialized Countries. Health Aff. 2000, 19, 191-203. [CrossRef]

2. EuroHealthNet. Europeans of Retirement Age: Chronic Diseases and Economic Activity. EU Report. Available online: http:/ / www.healthyageing.eu/news/rivm-report-europeans-retirement-age-chronicdiseases-and-economic-activity (accessed on 9 September 2018).

3. Sambamoorthi, U.; Tan, X.; Deb, A. Multiple chronic conditions and healthcare costs among adults. Expert Rev. Pharmacoecon. Outcomes Res. 2015, 15, 823-832. [CrossRef] [PubMed]

4. Chapman, K.R.; Mannino, D.M.; Soriano, J.B.; Vermeire, P.A.; Buist, A.S.; Thun, M.J.; Connell, C.; Jemal, A.; Lee, T.A.; Miravitlles, M.; et al. Epidemiology and costs of chronic obstructive pulmonary disease. Eur. Respir. J. 2006, 27, 188-207. [CrossRef] [PubMed]

5. European Heart Network. European Cardiovascular Disease Statistics 2017. Available online: http://www. ehnheart.org/images/CVD-statistics-report-August-2017.pdf (accessed on 18 October 2018).

6. Go, A.S.; Mozaffarian, D.; Roger, V.L.; Benjamin, E.J.; Berry, J.D.; Borden, W.B.; Bravata, D.M.; Dai, S.; Ford, E.S.; Fox, C.S.; et al. Heart disease and stroke statistics-2013 update: A report from the American Heart Association. Circulation 2013, 127, e6-e245. [CrossRef]

7. Dexter, P.R.; Miller, D.K.; Clarck, D.O.; Weiner, M.; Harris, L.E.; Livin, L.; Myers, I.; Shaw, D.; Blue, L.A.; Kunzer, J.; et al. Preparing for an Aging Population and Improving Chronic Disease Management. In Proceedings of the American Medical Informatics Association (AMIA) Annual Symposium, Washington, DC, USA, 13 November 2010; AMIA: Bethesda, MD, USA, 2010; pp. 162-166.

8. Coleman, K.; Austin, B.T.; Brach, C.; Wagner, E.H. Evidence on The Chronic Care Model in The New Millennium. Health Aff. 2009, 28, 75-85. [CrossRef] [PubMed]

9. Barr, V.J.; Robinson, S.; Marin-Link, B.; Underhill, L.; Dotts, A.; Ravensdale, D.; Salivaras, S. The Expanded Chronic Care Model: An Integration of Concepts and Strategies from Population Health Promotion and the Chronic Care Model. Healthc. Q. 2003, 7, 73-82. [CrossRef]

10. Bodenheimer, T.; Wagner, E.H.; Grumbach, K. Improving primary care for patients with chronic illness. JAMA 2002, 288, 1775-1779. [CrossRef] [PubMed] 
11. Leal, F.; Morais, C.; Pimenta, R. Cross-cultural adaptation and validation of the assessment of chronic illness care (ACIC): Chronic care model—Information for chronic disease management. In Proceedings of the IEEE CISTI, Barcelona, Spain, 14 August 2014; pp. 1-7.

12. Donati, M.; Celli, A.; Ruiu, A.; Saponara, S.; Fanucci, L. A Telemedicine Service Platform Exploiting BT/BLE Wireless Sensors for Remote Monitoring of Chronic Patients. In Proceedings of the 7th International Conference on Modern Circuits and Systems Technologies (MOCAST), Thessaloniki, Greece, 7-9 May 2018; IEEE: Piscataway, NJ, USA, 2018.

13. Daw, W.; Kingshott, R.; Scott, A.; Saatchi, R.; Elphick, H. Development of the 'BreathEasy' contactless portable respiratory rate monitor (CPRM). Eur. Respir. J. 2015, 46, PA1583. [CrossRef]

14. Fanucci, L.; Benini, A.; Donati, M.; Iacopetti, F. Self Electrocardiogram Acquisition Device. WO2015083036 A1, 11 June 2015.

15. Fanucci, L.; Saponara, S.; Bacchillone, T.; Donati, M.; Barba, P.; Sanchez-Tato, I.; Carmona, C. Sensing Devices and Sensor Signal Processing for Remote Monitoring of Vital Signs in CHF Patients. IEEE Trans. Instrum. Meas. 2013, 62, 553-569. [CrossRef]

16. Abbate, S.; Avvenuti, M.; Bonatesta, F.; Cola, G.; Corsini, P.; Vecchio, A. A smartphone-based fall detection system. Pervasive Mob. Comp. 2012, 8, 883-899. [CrossRef]

17. Li, J.-F.; Wang, Q.-H.; Liu, X.-M.; Cao, S.; Liu, F.L. Pedestrian Dead Reckoning System Integrating Low-Cost MEMS Inertial Sensors and GPS Receiver. J. Eng. Sci. Technol. Rev. 2014, 7, 197-203. [CrossRef]

18. Cleland, I.; Kikhia, B.; Nugent, C.; Boytsov, A.; Hallberg, J.; Synnes, K.; McClean, S.; Finlay, D. Optimal placement of accelerometers for the detection of everyday activities. Sensors 2013, 13, 9183-9200. [CrossRef] [PubMed]

19. Yadav, J.; Rani, A.; Singh, V.; Murari, B.M. Near-infrared LED based non-invasive blood glucose sensor. In Proceedings of the International Conference on Signal Processing and Integrated Networks (SPIN), Noida, India, 20-21 February 2014; IEEE: Piscataway, NJ, USA, 2014; pp. 591-594.

20. Gan, K.B.; Yahyavi, E.S.; Ismail, M. Contactless respiration rate measurement using optical method and empirical mode decomposition. J. Technol. Health Care 2016, 24, 761-768. [CrossRef] [PubMed]

21. Rieger, R.; Rif'an, M. Integrated ExG, vibration and temperature measurement front-end for wireless sensing. IEEE Trans. Circuits Syst. I 2018, 65, 2422-2430. [CrossRef]

22. Fang, D.; Hu, J.; Wei, X.; Shao, H.; Luo, Y. A Smart Phone Healthcare Monitoring System for Oxygen Saturation and Heart Rate. In Proceedings of the International Conference on Cyber-Enabled Distributed Computing and Knowledge Discovery, Shanghai, China, 13-15 October 2014; IEEE: Piscataway, NJ, USA, 2014; pp. 245-247.

23. Lutze, R.; Waldhor, K.A. Smartwatch Software Architecture for Health Hazard Handling for Elderly People. In Proceedings of the International Conference on Healthcare Informatics, Dallas, TX, USA, 21-23 October 2015; IEEE: Piscataway, NJ, USA, 2015; pp. 356-361.

24. Masella, C.; Zanaboni, P.; Borghi, G.; Castelli, A.; Marzegalli, M.; Tridico, C. Introduction of a telemonitoring service for patients affected by chronic heart failure. In Proceedings of the 11th International Conference on e-Health Networking, Applications and Services, Sydney, Australia, 16-18 December 2009; pp. 138-145.

25. Zhou, F.; Yang, H.-I.; Álamo, J.M.R.; Wong, J.S.; Chang, C.K. Mobile personal health care system for patients with diabetes. In Lecture Notes in Computer Science; Lee, Y., Bien, Z.Z., Mokhtari, M., Kim, J.T., Park, M., Kim, J., Lee, H., Khalil, I., Eds.; Springer: Berlin, Germany, 2011; pp. 94-101. ISBN 978-3-642-13778-5.

26. V-Patch Medical System. Available online: http://www.vpatchmedical.com (accessed on 20 November 2018).

27. Isansys Lifecare. Available online: http:/ / www.isansys.com (accessed on 20 November 2018).

28. Docobo. Available online: http:/ / www.docobo.co.uk (accessed on 20 November 2018).

29. Donati, M.; Bacchillone, T.; Fanucci, L.; Saponara, S.; Costalli, F. Operating protocol and networking issues of a telemedicine platform integrating from wireless home sensors to the hospital information system. J. Comp. Netw. Commun. 2013, 2013, 781620. [CrossRef]

30. Stankevich, E.; Paramonov, I. Using Bluetooth on Android Platform for mHealth Development. In Proceedings of the 10th Conference of FRUCT Association, Tampere, Finland, 8-10 November 2011; pp. 140-145. 
31. Bacchillone, T.; Donati, M.; Saponara, S.; Fanucci, L. A flexible home gateway system for telecare of patients affected by chronic heart failure. In Proceedings of the 5th International Symposium on Medical Information and Communication Technology, Montreux, Switzerland, 27-30 March 2011; IEEE: Piscataway, NJ, USA, 2011; pp. 139-142.

32. Calamai, R.; Giarre, L. Enabling primary and specialist care interoperability through HL7 CDA release 2 and the Chronic Care Model: An Italian case Study. IEEE Trans. Syst. Man Cybern. 2012, 42, 1364-1384. [CrossRef]

33. de Miguel-Bilbao, S.; Aguirre, E.; Lopez Iturri, P.; Azpilicueta, L.; Roldán, J.; Falcone, F.; Ramos, V. Evaluation of Electromagnetic Interference and Exposure Assessment from s-Health Solutions Based on Wi-Fi Devices. BioMed Res. Int. 2015, 2015, 784362. [CrossRef] [PubMed]

34. Golmie, N.; Rebala, O.; Chevrollier, N. Bluetooth adaptive frequency hopping and scheduling. In Proceedings of the IEEE MILCOM Conference, Boston, MA, USA, 13-16 October 2003; IEEE: Piscataway, NJ, USA, 2003. [CrossRef]

35. Health Level Seven International Site. Available online: http://www.hl7.org/implement/standards/ product_brief.cfm?product_id=7 (accessed on 22 December 2018).

(C) 2019 by the authors. Licensee MDPI, Basel, Switzerland. This article is an open access article distributed under the terms and conditions of the Creative Commons Attribution (CC BY) license (http:/ / creativecommons.org/licenses/by/4.0/). 